УДК 519.21

\title{
System Mathematical Models for the Formation of Signals and Synchronous Interference with the Use of Pulsed Non-explosive Seismic Sources
}

\author{
Georgy Ya. Shaidurov* \\ Danil S. Kudinov ${ }^{\dagger}$ \\ Siberian Federal University \\ Kirenskogo, 26, Krasnoyarsk, 660074
}

Russia

Received 06.08.2015, received in revised form 07.09.2015, accepted 06.10.2015

\begin{abstract}
The theory of linear systems is applied to simulate and describe signals and synchronous noise registered by seismic sensors. An algorithm is proposed to reduce the effect of the inhomogeneous upper layer and of regular interference associated with the low velocity zone.

Mathematical models of synchronous noise are given with consideration for their randomly changing parameters.

The materials from this paper can be helpful in developing algorithms to process data acquired primarily by nonexplosive pulsed and vibrational seismic survey technologies.
\end{abstract}

Keywords: mathematical formulation, signals, noise, exploration seismology, electromagnetics, wave, pulsed nonexplosive sources.

DOI: $10.17516 / 1997-1397-2015-8-442-453$

\section{Introduction}

The fundamental physical processes initiated in geological environment by induced seismic vibrations, including those generated by nonexplosive sources, according to the current theory of seismic surveying can be simulated by a linear model described by a set of differential equations with constant coefficients $[1,2,3,4,5,6]$. Assuming this approach, the characteristics that are sufficient to describe the model embrace the transfer function $(T F)$, amplitude-frequency characteristic $(A F C)$, phase-frequency characteristic $(P F C)$, and transient response $(T R)$ or pulse characteristic $(P C)$, all being mathematically uniquely interdependent via integral Fourier and Laplace transforms [3]. The excitation transfer function of seismic vibrations and propagation of the latter in a geological structure can roughly be described by the characteristics of an oscillatory circuit (Shneerson and Mayorov, 1988) [7, 8,9].

\section{Linear model of seismic signals formation}

Fig. 1 illustrates a simplified linear pattern of formation of a seismic signal shown as a sequence of individual stages of transfer of the excitation signal $I(t)$ generated by a nonexplosive source $[10,11,12,13]$

The transmitting properties of the "source-ground surface" system are modeled by the pulse characteristic $(P C) F_{G}(p)$ and its counterpart $T R F_{G}(p)$, where $t$ is the current time, $P=C \pm j$

\footnotetext{
*GShaidurov@sfu-kras.ru

$\dagger$ KudinovDanil@yandex.ru

(C) Siberian Federal University. All rights reserved
} 


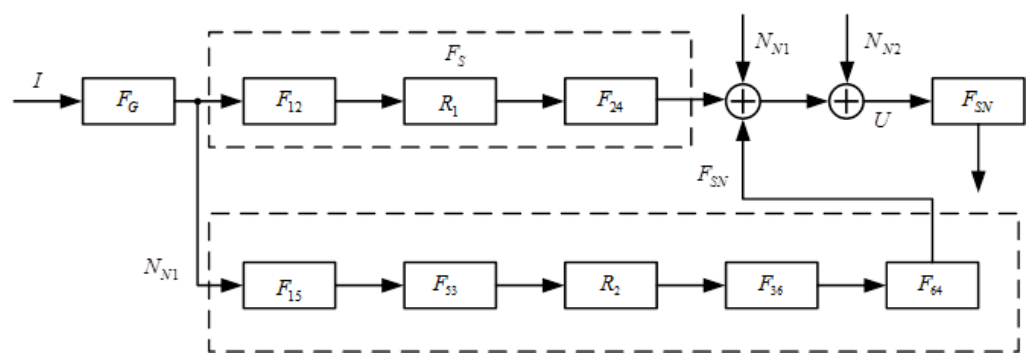

Fig. 1. A linear system model of signal formation in a two-layer model of seismic cross-section

is the complex frequency. The transfer properties of formation of a useful signal are represented by the block with the pair $F_{S}(t), F_{S}(p)$. The $F_{S N}$ block is responsible for synchronous noise $(S N)$ produced by shear, refracted or re-reflected vibrations propagating in the upper layers of a geological cross-section during seismic surveying.

The transfer function $F_{S N}(p)$ describes the linear section of a seismic array, i.e. it incorporates the seismic sensor itself as well as primary processing of signals during signal recording such as for example digital filtration.

Since the frequency band of any seismic detector is considerably broader than the frequency band emitted by a source of probing signals $(P S)$, in our further discussion $F S N(p)$ will be understood as $T F$ of a receiver carrying out primary signal processing before recording or secondary signal processing performed by an interpreter $[14,15]$.

Note that the seismic sensor is a vibrational system with $T F$ corresponding to the first derivative of dynamic displacement of the soil in which the seismic sensor is embedded.

SN signals arrive at the seismic receiver simultaneously with the useful signal reflected from the target zone at a chosen depth. Since SN signals are generated by the same common source of seismic waves, increasing the power of the latter is not going to affect the ratio $q=\frac{S}{S N}$, therefore when optimizing the ratio $\frac{S}{S N+N}$, where noise comprises microseisms and noises not correlated with the probing signal, we need to be specific about the kind of interference we seek to eliminate.

In seismic surveying, a conventional approach to improve the $S / N$ ratio is to accumulate signal under repeated excitation of the geological cross-section. Depending on spectral composition, adequate noise elimination techniques should be chosen to meet the task. Note that the signal accumulation method is efficient to eliminate non-correlated broad band noise.

In Fig.1 microseisms are represented by the noise spectral power density $N_{N 1}$. The intrinsic noise of the seismic sensor is represented by the spectral density $N_{N 2}$. Considering the above points, a combination of the useful signal, SN and other noises can be written as a functional equation:

$$
U(t, p)=I(t, p) \otimes F_{G} \otimes F_{S} \otimes F_{S I}+I(t, p) \otimes F_{G} \otimes F_{N} \otimes F_{S N}+\left[\overline{N_{1} \otimes F_{S N}^{2}}\right]^{\frac{1}{2}}+\left[\overline{N_{2} \otimes F_{S N}^{2}}\right]^{\frac{1}{2}}
$$

In this equation, the sign $\otimes$ stands for functional multiplication and the bar over the last two terms denotes averaging over time or over frequency. The most general criterion for optimization of seismic surveying in the statistical detection theory, which is applied in particular in radiolocation, is the likelihood ratio [16]:

$$
\frac{\lambda\left(U_{s}, U_{n}\right)}{\Lambda\left(U_{n}\right)} \geqslant \frac{2 E}{N},
$$


where $\Lambda(U S, U N)$ is the signal and noise distribution function obtained after each subsequent seismic event; $\Lambda\left(U_{N}\right)$ is the noise distribution function; $\mathrm{E}$ is the energy of the useful signal, and $N_{N}$ is the noise spectral power density.

It is known that all information about signals and noise is concentrated in the probability distribution function of the observable realization. Criterion (2) under a normal law of noise and interference distribution is equivalent in power to the signal-to-interference plus noise relation:

$$
q=\frac{\left[I \otimes F_{G} \otimes F_{S} \times F_{S N}\right]^{2}}{\left[I \otimes F_{G} \otimes F_{S} \times F_{S N}\right]^{2}+N_{N 1} F_{S N}^{2}+\overline{N_{N 2} F_{S N}}} .
$$

When the magnitude of $S N$ is small compared to noise, increasing the number of impacts from the pulse source by $n$ times in order to improve the signal-to-noise ratio yields:

$$
q=\frac{n\left[I \otimes F_{G} \otimes F_{S}\right]^{2}}{N_{N 1}+N_{N 2}} .
$$

In the case of $\frac{S N}{N}>>1$, Formula (3) transforms into the expression:

$$
q=\left(\frac{F_{S}}{F_{N}}\right)^{2}
$$

That is, the $\frac{S}{S N}$ ratio observed at the seismic sensor is not affected by the impact repetition rate but solely depends on the TF ratio between the signal and synchronous noise.

Thus, aiming at minimization of the number of impacts, one has to acquire information on the signal-to-noise ratio after having eliminated synchronous noise by one of the available techniques.

\section{Signals formation in the two-layer and three-layer models of a seismic cross-section}

Let us estimate the signal-to-noise ratio for a two-layer model of a seismic cross-section. Find $T F$ contained in Equations (1) and (3) using Fig. 2. We first dwell on the two-layer model of seismic cross-section shown in Fig. 2.

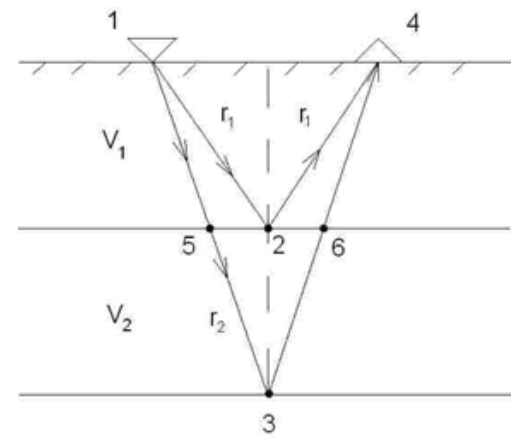

Fig. 2. Signal formation in a two-layer model of seismic cross-section

When a pulsed source acts on a seismic cross-section the sound signal, having traveled distance $r_{1}$, is reflected from the first boundary and enters the seismic sensor at point 4 . We will assume 
the seismic sensor to be in close vicinity of the source so that the path $2 r_{1}$ is a primary reflection. Hence the signal arrival delay time at these lengths will be estimated as

$$
t_{1}=\frac{2 r_{1}}{V_{1}}
$$

and

$$
t_{2}=\frac{2 r_{1}}{V_{1}}+\frac{2\left(r_{1}-r_{2}\right)}{V_{2}},
$$

$V_{1}$ and $V_{2}$ being the propagation velocities. Provided $r_{2}>>r_{1}$, we assume the distance between points 1,5 and 2,6 to be approximately equal to $r_{1}$.

Reflection coefficients at points 2 and 3 are taken as scalars $R_{1}$ and $R_{2}$. The output signal from the seismic sensor for this model will be equal to

$$
U=\left\{I F_{G} F_{12} R_{1} F_{24}+I F_{g} F_{15} F_{53} R_{2} F_{36} F_{64}\right\} F_{S N}+\left[\overline{N_{N} F_{S N}^{2}}\right]^{\frac{1}{2}} .
$$

Subject to condition (7) satisfied, equation (8) can be cast in the form

$$
U=\left\{I F_{G} F_{12} R_{1} F_{24}+I F_{g} F_{12} F_{53} R_{2} F_{36} F_{64}\right\} F_{S N}+\left[\overline{N_{N} F_{S N}^{2}}\right]^{\frac{1}{2}}=U_{S}+U_{S N}+U_{N} .
$$

The first arrival event at the seismic sensor in this case generates a synchronous noise signal while the useful signal, having been reflected from the second layer at point 3 , is detected as a second arrival with t2 delay. We can therefore, using equation (9), extract an uncontaminated useful signal by dividing the second term in braces in (9) by the first one, which yields:

$$
F_{56}=F_{53} \frac{R_{2}}{R_{1}} F_{36}
$$

i.e. we obtain an uncontaminated $T F$ of the second layer, wherefrom it follows that in terms of power the sought signal-to-noise ratio will be equal to

$$
q=\left(\frac{S}{N}\right)_{p}=\frac{R_{2}}{R_{1}} \cdot \frac{\int_{\omega_{1}}^{\omega_{2}} I_{2}(j \omega) F_{53}^{2}(j \omega) F_{36}^{2}(j \omega) d \omega}{N_{N}\left(\omega_{2}-\omega_{1}\right)}
$$

where $\left[\omega_{1}, \omega_{2}\right]$ is the signal frequency interval; $\left(\omega_{1}-\omega_{2}\right)$ is the operating frequency band; $N_{N}$ is the spectral power density of non-correlated noise.

The ratio $\frac{R_{2}}{R_{1}}$ can be found as a modulus ratio between signal amplitudes at delay times $t_{1}$ and $t_{2}$, i.e. $\frac{R_{2}}{R_{1}}=\frac{\left[U\left(t_{2}\right)\right]}{\left[U\left(t_{1}\right)\right]}$.

Introducing a third layer or any further layers with clearly defined boundaries into the model in Fig. 2 will not make any difference as we are talking about distinguishing a useful signal that arrives from the maximum possible depth against a synchronous interfering signal coming from the first inhomogeneous layer. An algorithm to determine the signal-to-noise ratio thus comes down to acquiring spectral characteristics of signals coming from the nearest to the source seismic sensors and then finding the useful signal component by diving spectral characteristics of the latest detected signal by those of the first arrived signal. The useful signal power is found by integrating the obtained division result over frequency. Relation between the useful signal power and the noise power in the signal band determines the sought signal-to-noise ratio.

Physically, this algorithm enables us to eliminate interference of the inhomogeneous upper layer with the useful signal, considering the above made assumptions. 
Note that $\frac{1}{2 \pi} N_{N}\left(\omega_{1}-\omega_{2}\right)=P_{N}=\sigma_{N}^{2}$, i.e. the denominator in (11) can be found through noise dispersion in the receiver transmission band. The number of layers in a geological crosssection increases with offset of seismic sensors from the excitation source, therefore algorithm (11) requires modification to allow for reverberation and refracted waves emerging in near-surface layers of the earth.

We will use the absorption factor $e^{-\alpha(\omega) r i}$ to allow for frequency-dependent absorption of seismic waves and factor $e^{p r i}$ to account for delayed seismic waves, where $p= \pm j \omega$ is the complex frequency and is the time of delay over the signal propagation path.

Fig. 3 shows a three-layer cross-section and ray paths of the observed signal formation at the output of two adjacent seismic sensors.

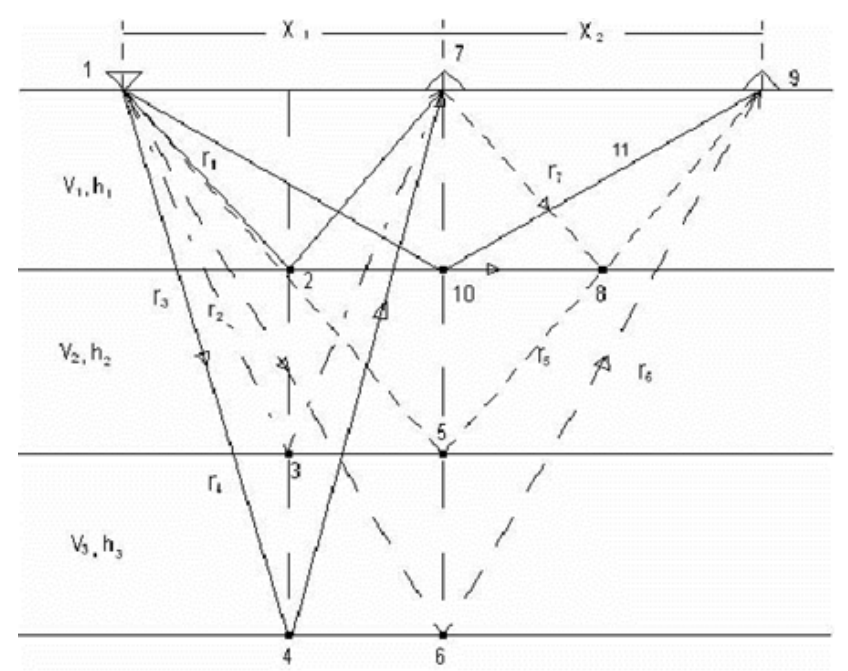

Fig. 3. Signal formation in a three-layer model

Synchronous noise in the form of overlapping reverberation and refracted waves is generated by the first layer.

The seismic sensor nearest to the source at point 7 receives only re-reflected signals of $P$-waves and $S$-waves from all layers whereas the second seismic sensor at an arbitrary offset $x_{2}$ from the excitation source detects synchronous noise produced by the first layer as well as useful signals of the first reflection.

We will only be interested in $P$-waves.

Write down the transfer functions of the model in Fig. 3 as follows:

$$
\begin{gathered}
F_{12} F_{27}=F_{17} e^{-p \tau_{2}}, \quad \tau_{2}=\frac{2 r_{1}}{V_{1}}, \\
F_{13} F_{37}=F_{137} e^{-p \tau}, \quad \tau_{2}=2\left(\frac{r_{1}}{V_{1}}+\frac{r_{3}-r_{1}}{V_{2}}\right), \\
F_{14} F_{47}=F_{147} e^{-p \tau_{4}}, \quad \tau_{4}=\frac{r_{1}}{V_{1}}+\frac{r_{3}-r_{1}}{V_{2}}+\frac{R_{34}}{V_{3}} .
\end{gathered}
$$

The reverberation interference caused by double reflection from the first layer is

$$
F_{12} F_{27} F_{78} F_{89}=F e^{-p \tau_{219}}, \quad \tau_{219}=\frac{4 r_{1}}{V_{1}} .
$$


The transfer function of reverberation interference of triple reflection from the first layer can be written as:

$$
F_{3 p 19}=F_{12}^{6} e^{-p \tau_{319}}, \quad \tau_{319}=\frac{6 r_{1}}{V_{1}}
$$

1-10 are the conjugation and receiving points; 11 is the wave refracted by the first layer; $V_{i}$, $h_{i}$ are the signal propagation velocities and layer thicknesses, respectively; $r_{i}$ is the signal path length in the layer; $x_{1}, x_{2}$ is the distance between the source and seismic sensors. An equivalent system model of the problem under discussion is given in Fig. 4.

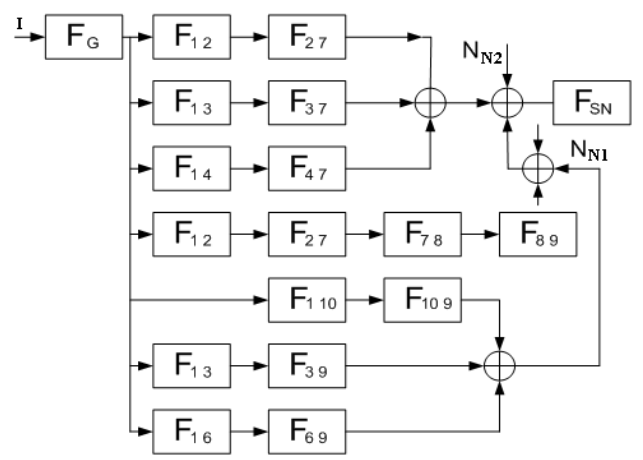

Fig. 4. System model for a three-layer model

Define $T F$ of the refracted wave signal in the first layer approximately as:

$$
F_{N 19}=F_{12}^{2} F_{22} e^{-p \tau_{N 1}}, \quad \tau_{N 1}=\frac{2 r_{1}}{V_{1}}+\frac{x_{2}}{V_{N}},
$$

where $\Phi_{22}$ and $V_{N}$ are the transfer function and propagation velocity of the refracted wave, respectively. All the rest $T F$ are found from Fig. 3 in a similar way.

We will be interested in a method to separate a useful signal of the first reflection from an arbitrary layer of reverberation waves and the first layer of refracted waves using information acquired from the receiver nearest to the seismic source and the one offset at $x_{2}$.

If the intensity of the refracted wave is small compared to the reverberation $(R B)$ wave, the $R B$ interference can be recovered through Exp. (16).

From Fig. 3 one can estimate multiplicity of reflection for the $R B$ interference.

The condition for $\mathrm{RB}$ noise to interfere with the useful signal of the $i$-th layer is

$$
\tau_{R B}=\tau_{i},
$$

where, for instance, for the second layer signal we have

$$
\tau_{i}=\tau_{5}=\frac{2 r_{5}}{V_{2}} .
$$

For the third layer we have

$$
\tau_{i}=\tau_{6}=\frac{2 r_{6}}{V_{3}}
$$

where $V_{2}$ and $V_{3}$ are the effective propagation velocities of signals traveling to reflection points 5 and 6 , adjusted for the propagation velocities in the first and the second layer.

The $R B$ interference delay is

$$
\tau_{R B}=\frac{2 k r_{1}}{V_{1}}
$$


Here $k$ is the multiplicity of reflection that can be defined as the relation $\frac{\tau_{R B}}{\tau_{i}}$. So for the second layer we have

$$
\frac{\tau_{R B}}{\tau_{5}}=\frac{2 k r_{1} V_{2}}{V_{1} \cdot 2 r_{5}} .
$$

Since the signal delay times $\tau_{1}=\frac{2 r_{1}}{V_{1}}$ and $\tau_{5}=\frac{2 r_{5}}{V_{2}}$ are known from detection results, the multiplicity factor can be calculated straightforward from (22). The presence of $R B$ interference is determined by the criterion $k=1,3,5 \ldots$, i.e. by odd natural numbers.

If ratio (22) for the detected signal meets this criterion, then the useful signal is contaminated by $R B$ interference.

In this case, relations of the type such as (15) and (16) are used to synthesize an $R B$ interference signal based on $T F$ data of the first layer $\Phi_{12}$, derived from the signal of the nearby seismic sensor at point 7 . The so recovered RB interference is then subtracted from the useful signal reflected from a layer of interest. Under pulsed excitation of a medium, the reflection coefficient of the medium is frequency dependent, which results in the pulse stretching in time. This is associated with its high-frequency spectrum being limited as seismic waves penetrate deeper into the geological structure. Fig. 5 depicts the temporal shape of signal pulses for various distances from the source (Gurvich and Boganin, 1980).

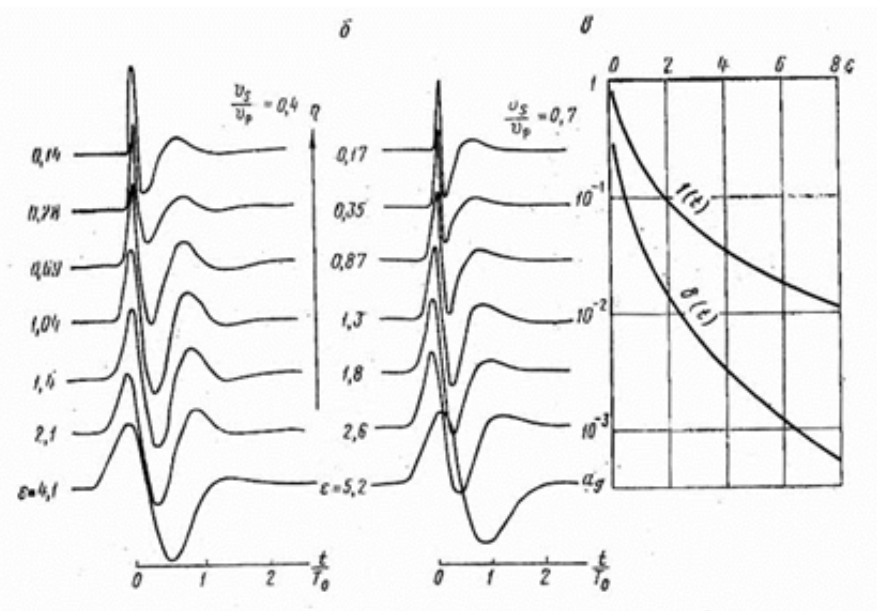

Fig. 5. Changes in the shape of the pulsed signal of a seismic wave in an absorbing medium depending on distance

As has been noticed in (Shneerson and Mayorov, 1988), an increased absorption decrement is frequently observed in gas and oil fields. Absorption of seismic energy particularly in gas-bearing strata may be several times stronger than in water-saturated beds.

Many papers report emergence of converted waves over oil and gas reservoirs and the preferred registration of $\mathrm{P}$-waves for the purpose of their direct search.

\section{Stochastic models of description of the observed signals and noises}

We now turn to a mathematical model of synchronous noise, considering randomness of the noise parameters. Conventionally, in order to extract a useful signal from noise in seismic surveying practice, the linear filtration method is predominantly used where useful signals and 
noise of all sorts are distinguished by their frequency spectra. Spectral characteristics of various types of waves, including microseisms, are shown in Fig. 6 [1,2].

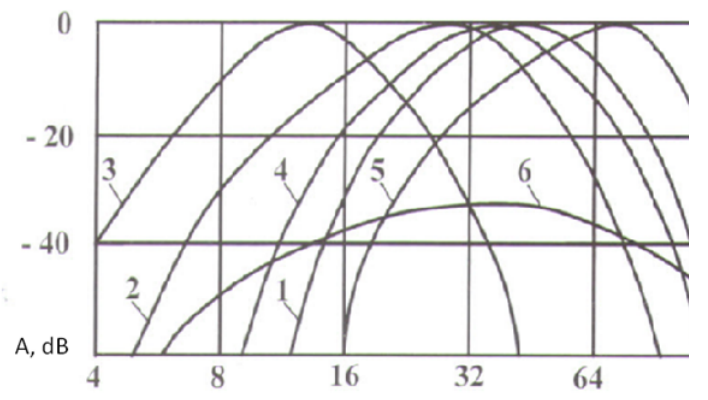

Fig. 6. Compare relative amplitude spectra registered by reflection techniques for various seismic waves: 1 is $P$-waves; 2 is $S$-waves; 3 is surface waves; 4 is multiple $P$-waves; 5 is reverberation interference in shallow water; 6 is microseisms

As is evident from the picture, a pronounced difference in the spectra is observed between useful signals and low-frequency surface noise originating in the low-velocity zone $(L V Z)$ in the upper part of the geological cross-section adjacent to the free soil surface. The $P$-wave signals are somewhat frequency-shifted downward (by about $8 \mathrm{~Hz}$ ) and a frequency shift upward by the same $8 \mathrm{~Hz}$ is observed for multiple wave signals or reverberations. Noise not correlated with the signal (microseisms) features a wide spectrum ranging from $5 \mathrm{~Hz}$ to100 Hz and above. The dynamic range of signals and noise can be as large as $100 \div 120 \mathrm{~dB}$, which substantially complicates the algorithms of noise elimination.

As mentioned above, we use the term "synchronous noise" rather than the conventional seismic terminology (regular, irregular, etc. interference) to refer to the interference whose rate depends on the power of the excitation source. We believe that this is a more comprehensive term that indicates the origin of interference (Shaidurov, 1999) [9].

Because of the difference in the methods of elimination between regular and irregular (statistic) components of $S N$, we will represent them as a combination of two terms (23)

$$
U_{S N}(t)=\bar{U}_{S N}(t)+\tilde{U}_{N}(t)
$$

where the second term accounts for random fluctuations of the $S N$ amplitude.

Express (23) through the transfer function $(T F)$ of synchronous noise $F_{N}(\Lambda, \partial)$ :

$$
U_{S N}(t)=\frac{1}{2 \pi} \int_{-\infty}^{+\infty} I_{u}(p) \cdot F_{G}(p) \cdot F_{S N}\left(\lambda_{1} p\right) \cdot e^{p t} d p
$$

where $\Lambda$ is the observed $S N$ parameter containing, as in (23), two components:

$$
\Lambda=\bar{\Lambda}+\tilde{\Lambda}
$$

Here $\bar{\Lambda}$ is the deterministic component of the parameter and $\tilde{\Lambda}$ is the random component.

Expanding the transfer function of $S N$ in Taylor series about the small parameter $\bar{\Lambda}$ and separating the linear part we can write down the random $S N$ component as a time function,

$$
\tilde{U}_{S N}(t)=\Delta \Lambda \int_{0}^{\infty} I(t) F_{G}(t-T) d t \int_{0}^{\infty} \frac{\partial}{\partial \Lambda} \cdot F_{S N}(\Lambda, t, \tau) d \tau
$$

where $\Delta \Lambda$ is the increment of parameter $\Lambda$. 
The validity of the small parameter method is subject to specific requirements.

In particular, if the regular $S N$ component (multiple waves being the case) changes little across the seismic profile and $\Delta \Lambda<<\Lambda$, then in this case (26) holds true.

Write the regular $S N$ component as

$$
\bar{U}_{S N}(t)=\int_{0}^{\infty} I(t) F_{G}(t-\tau) d t \int_{0}^{\infty} \bar{F}_{S N}(\bar{\Lambda}, t, \tau) d \tau
$$

Now cast expression (27) in a somewhat different form:

$$
\bar{U}_{S N}(t)=\int_{0}^{\infty} I(t) d t \int_{0}^{\infty} F_{G}(\tau) \bar{F}_{S N}(\bar{\Lambda}, t, \tau) d \tau .
$$

Expression

$$
B_{S N} \int_{0}^{\infty} F_{G}(\tau) \bar{F}_{S N}(\bar{\Lambda}, t, \tau) d \tau
$$

is a mutual correlation function between the source and $S N$ transfer functions.

Quantities (26) and (27) being statistically independent, we can define the correlation function of synchronous noise as:

$$
B(m, \tau)=G_{\Lambda}^{2} R_{\Lambda}(m) \tilde{B}_{S N}(\tau)+M_{\Lambda}^{2}
$$

where

$$
R_{\Lambda}(m)=\frac{\Delta \tilde{\Lambda}(n) \cdot \Delta \Lambda(n+m)}{G_{\Lambda}^{2}}
$$

is the correlation function of a random $S N$ parameter; $G_{\Lambda}^{2}$ is the amplitude dispersion of parameter $\Lambda ; n$ is the seismic sensor number; $m$ is the spatial shift in seismic sensor numbers; $M_{\Lambda}^{2}$ is the squared constant component of $\Lambda$ parameter.

$$
\tilde{B}_{S N}(\tau)=\int_{0}^{\infty} \frac{\partial}{\partial \Lambda} F_{S N}(\Lambda, t) \frac{\partial}{\partial \Lambda} F_{S N}(\Lambda, \tau-t) d \tau .
$$

The correlation function of the deterministic part of synchronous noise is written as:

$$
B_{S N}(\tau)=\int_{0}^{\infty} F_{S N}(\bar{\Lambda}, t) F_{S N}(\bar{\Lambda}, \tau-t) d \tau .
$$

Write the energy spectra of $S N$ in the following form:

$$
\begin{gathered}
F_{S N}(\omega)=\left[\bar{F}_{S N}(\omega)+\tilde{F}_{S N}(\omega)\right] I_{u}^{2}(\omega) F_{G}^{2}(\omega), \\
\tilde{F}_{S N}(\omega) \frac{2}{T} \sigma_{\Lambda}^{2} G_{1}(\omega)^{2} F_{\lambda}(\omega) F_{G}^{2}(\omega), \\
G_{1}(\omega)=\int_{0}^{\infty} \frac{\partial}{\partial \Lambda} F_{S N}(\Lambda, t) e^{j \omega t} d t .
\end{gathered}
$$

In these expressions, $I_{u}^{2} \hat{O}_{G}^{2}(\omega)$ is the energy spectrum of the excitation wave (probe signal)

$$
F_{\Lambda}(\omega)=\frac{1}{T} \int_{0}^{\infty} \tilde{R}_{\Lambda}(\tau) e^{j \omega \tau} d \tau
$$

$T$ is the observation interval.

Consider now sources of origin of interference. In Eastern Siberia and other similar regions of the world, the major problem in seismic exploration is posed by inhomogeneity of the near-surface layer, 500 to $1000 \mathrm{~m}$ thick, where formation of a seismic signal is stochastic. 
Waves of actually any type are possible to occur: pressure, shear, conversion, Rayleigh, Lamb, and other waves. The top section of this layer (up to $100 \mathrm{~m}$ in thickness) is usually classified as a low-velocity zone $(L V Z)$ in which the $P$-wave velocity is substantially smaller than in the deeper down layers. Raypaths of $P$-waves because of $L V Z$ are severely bent at the bedrock $/ L V Z$ interface. Inside $L V Z, P$-wave raypaths deviate toward vertical direction. The observed seismic signals from $L V Z$ are frequency-shifted downwards because of absorption of high-frequency spectral components in the upper layers. As the offset from the source grows, attenuation of the wave intensity occurs faster than in the weakly absorbing medium due to a strong absorption of the wave energy in $L V Z$.

The bottom boundary of $L V Z$ for $S$-waves often appears to be not so much pronounced because velocity $V_{S}$ is less dependent on water saturation of the strata. Therefore the upper part of a stratigraphic cross-section is less inhomogeneous for $S$-waves than for $P$-waves. As noted in (Bondarev, 2007), there are virtually no low velocity zones in permafrost areas [1].

he wavelength $\lambda_{p}$ in the medium is shorter as a result of lower wave velocities in $L V Z$, which makes measurements more sensitive to the heterogeneous structure of layers. Being slower, the signals of regular synchronous noise that arrive from inhomogeneous upper layers overlap in time with useful signals arriving from informative deeper layers. So their principal distinguishing feature may be the difference in their spectra.

Unfortunately, the amplitude of synchronous noise from upper layers may be tens to hundreds times stronger than the level of useful signals. If this is the case, it would be necessary to apply nonlinear procedures where filter transmission coefficients are controlled to match a particular signal-to-noise ratio.

\section{Conclusion}

1. A linear system model has been synthesized to describe the formation of output seismic sensor signals and noise for seismic waves produced by a nonexplosive pulsed source.

2. A notion of synchronous noise has been introduced to embrace all kinds of regular and irregular interference generated by the source.

Signals that are not correlated with the source are treated as noise. For synchronous noise, the signal/noise relation is independent of the source power and can be controlled by selecting either a proper excitation pulse waveform in the soil immediately adjacent to the source baseplate or a proper current pulse shape in the electric source winding.

3. An algorithm has been devised to determine the signal-to-noise ratio based on data acquired from nearby seismic sensors offset from the source at a distance corresponding to a single reflection from the first inhomogeneous layer. This allows an optimum number of source impacts to be calculated.

4. An algorithm has been found to isolate multiple waves in a two-layer system. This helps solve the problem of compensation of the regular (deterministic) component of waves of that type.

5. A stochastic model has been proposed to describe synchronous noise. The model consists of a regular and a stochastic component. To describe the energy spectra of both components, formulae have been suggested based on the small parameter method.

6. Various sources of origin of synchronous noise as well as spectral and amplitude features of the latter have been analyzed. In Eastern Siberia, synchronous noise predominantly originates from low velocity zones and a geologically heterogeneous near-surface layer having the thickness up to $500 \div 1000 \mathrm{~m}$.

The paper has been financed under a federal complex project, П 218. 


\section{References}

[1] V.I.Bondarev, Seismic exploration, Yekaterinburg, 2007.

[2] G.N.Boganik, I.I.Gurvich, Seismic exploration, Publishing, 2006.

[3] G.Ya.Shaidurov, D.S.Kudinov, A.A.Shchitnikov, Pulsed non-explosive seismic sources with an electromagnetic drive, International Journal of Applied Engineering Research, 10(2015), no. 15,7 .

[4] E.Hilterman, Seismic amplitude interpretation, Tulsa, Penn Well Books, 2001.

[5] Oz.Ylimaz, Seismic Date Analysis, Tulsa, Society of Exploration Geophysicists, vol. 2, 2001.

[6] S.V.Goldin, Linear conversion of seismic signals, Moscow, Nedra 1974 (in Russian).

[7] I.S.Chichinin, Vibration emission of seismic waves, Moscow, Nedra, 1984 (in Russian).

[8] M.B.Shneerson, V.V.Majorov, Surface non-explosive seismic, Moscow. Nedra, 1988 (in Russian).

[9] G.Ya.Shaidurov, Pulsed electromagnetic system for search, Krasnoyarsk, KGTU, 1989 (in Russian).

[10] G.Ya.Shaidurov, V.A.Detkov, D.S.Kudinov, V.V.Suhotin, Formation of the signals and interference when using pulsed non-explosive sources of seismic waves, Kharkiv National University of Radio Electronics, Journal of Applied electronics 4(2012), 6.

[11] S.Deregowski, F.Rocca, Geometrical optics and wave theory for constant offset sections in layered MEDIA, Geophysical prospecting, 29(1981), 374-406

[12] O.Omoboya, J.J.S. de Figueiredo, L.Huang, Dyaur, R.R.Stewart, Experimental study of the influence of fluids on seismic azimuthal anisotropy, In review in Geophys. Prosp., 2013.

[13] M.E.Far, C.M.Sayers, L.Thomsen, D.Han, J.P.Castagna, Seismic characterization of naturally fractured reservoirs using amplitude versusoffset and azimuth analysis, Geophysical Prospecting, 61(2013), 427-447.

[14] V.A.Detkov, V.B.Kashkin, G.Ya.Shaidurov, On the potential resolution of the non-explosive seismic sources, Seismic technology, 4(2012), 5.

[15] D.F.Klaerbout, Seismic images of the Earth's crust, Moscow, Nedra, 1989 (in Russian).

[16] A.A.Nikitin, Theoretical foundations of geological information, Moscow, Nedra, 1986 (in Russian). 


\title{
Системные математические модели формирования сигналов и помех в импульсной невзрывной сейсморазведке
}

\section{Георгий Я. Шайдуров \\ Данил С. Кудинов}

\begin{abstract}
Теория линейных систем применяется для моделирования и описания сигналов и синхронного шума, зарегистрированных сейсмических датчиков. Предложен алгоритм для уменъшения влияния неоднородного верхнего слоя и регулярного вмешательства, связанного с зоной низкой скорости.

Математические модели синхронного шума даны с учетом своих случайно изменяющихся параметров.

Материалы из этой статъя могут быть полезны при разработке алгоритмов для обработки данных, полученных в первую очередъ невзрывоопасными импульсными и вибрационными технологиями сейсморазведки.

Ключевые слова: математическая постановка, сигналы, шум, сейсморазведка, электромагнетизм, волна, импульсные невзрывные источники.
\end{abstract}

